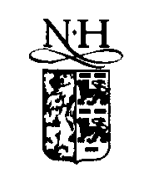

ELSEVIER
Nuclear Physics B (Proc. Suppl.) 112 (2002) 246-250

\title{
Jefferson Lab: Experimental Facilities, Upgrade Plans and Potential for Research Related to Neutrino-Nucleus Interactions
}

\author{
S. A. Wood ${ }^{\mathrm{a}}$ \\ aThomas Jefferson National Accelerator Facility, 12000 Jefferson Avenue, \\ Newport News, VA 23602, USA
}

The electron accelerator at Jefferson Lab (JLab) presently produces $100 \%$ duty factor beams at energies up to $6 \mathrm{GeV}$ for use in 3 endstations with a variety of detectors. Plans are presently being made for an energy upgrade to $12 \mathrm{GeV}$ along with new and upgraded experimental equipment. Possibilities of using Jlab facilities to make measurements of relevance to neutrino-nucleus interactions are discussed.

\section{Introduction}

The Thomas Jefferson National Accelerator Facility, more commonly known as Jefferson Lab or $\mathrm{JLab}$, operates a continuous duty factor electron accelerator with a peak energy of nearly $6 \mathrm{GeV}$. This energy is well suited to exploring the transition from nucleon based descriptions of nuclei to energies where PQCD describes nucleons and nuclei.

Electron beams at JLab are accelerated by two linacs in a racetrack design. Arcs of magnets circulate the beams up to five times through the linacs, supplying beam to 3 experimental areas, Halls A, B and C. All the halls can receive independenently controlled current and beam energy with the restriction that each hall's beam energy must be the sum the injector energy plus a multiple (up to five) of the energy per pass. A strained Gallium Arsenide source produces longitudinal beam polarizations of up to $80 \%$. Because of spin rotation in the accelerator arcs and the transport lines to the experimental areas, each hall can receive the maximal polarization only when the accelerator is set to certain "magic" energies. In each hall, the beam can be used as an electron beam or a photon beam. A photon beam can consist either of a Bremsstrahlung beam mixed with the primary electron beam, or in the case of Hall B, a pure tagged photon beam.

The three experimental halls are designed to accomodate a wide variety of types of experi- ments. Hall B contains the CEBAF Large Acceptance Spectrometer (CLAS) which can make measurements over broad kinematic ranges and with multiparticle final states. Higher resolution and luminosity measurements can be made in Hall's $\mathrm{A}$ and $\mathrm{C}$ which each contain a pair of moderate solid angle focusing spectrometers. In addition to the base equipment, additional detectors and spectrometers are frequently placed temporarily in the halls for specific experiments.

\section{Hall $A$ and $C$ Focusing Spectrometers}

Halls $\mathrm{A}$ and $\mathrm{C}$ both contain a pair of focusing spectrometers which share a number of common properties. These spectrometers have full focal plane tracking and can handle extended targets, such as liquid hydrogen, deuterium, helium, and polarized targets of at least $10 \mathrm{~cm}$ in length. The focal plane detector stacks can also distinguish between electrons, pions, kaons, and protons through a variety of particle ID techniques such as Time-of-Flight, $d E / d x$, threshold Cerenkov, and lead-glass shower. All the spectrometers have a target to focal plane flight path of approximately 25 meters except for the SOS (Short Orbit Spectrometer) in Hall C. The 10 meter flight path of the SOS makes it ideal for detection of low momentum pions and kaons.

A recoil proton polarimeter is included in one of the Hall A HRS (High Resolution Spectrometer) spectrometers and a similar polarimeter will 
be installed in the HMS (High Momentum Spectrometer) spectrometer in Hall C. The spectrometers in Hall $\mathrm{A}$ are being augmented with a superconducting septum magnet system that will allow for small scattering angles $\left(6^{\circ}\right.$ to $\left.12^{\circ}\right)$. The properties of these spectrometers are summarized in Table 1.

\begin{tabular}{|l||l||l|l|}
\hline \multicolumn{1}{|c||}{} & \multicolumn{1}{c||}{ Hall A } & \multicolumn{2}{c|}{ Hall C } \\
\hline & HRS $\times 2$ & HMS & SOS \\
\hline$\theta_{\min }$ & $12^{\circ}\left(6^{\circ}\right)$ & $10.5^{\circ}$ & $12^{\circ}$ \\
$\theta_{\max }$ & $160^{\circ}$ & $90^{\circ}$ & $135^{\circ}$ \\
$l$ & $25 \mathrm{~m}$ & $25 \mathrm{~m}$ & $10 \mathrm{~m}$ \\
$p_{\max }$ & $4 \mathrm{GeV} / c$ & $>7 \mathrm{GeV} / c$ & $2 \mathrm{GeV} / c$ \\
$\Delta p / p$ & $10 \%$ & $20 \%$ & $40 \%$ \\
$d p / p$ & $\sim 10^{-4}$ & $<10^{-3}$ & $<10^{-3}$ \\
$\vec{p}$ & Yes & Planned & \\
\hline
\end{tabular}

Table 1

Hall $\mathrm{A}$ and $\mathrm{C}$ spectrometer properties.

The Hall $\mathrm{A}$ and $\mathrm{C}$ spectrometers may be used for single arm measurements such as elastic or deep-inelastic $\left(e, e^{\prime}\right)$ or for coincidence measurements such as $\left(e, e^{\prime} p\right),\left(e, e^{\prime} \pi\right)$, or $\left(e, e^{\prime} K\right)$ using both the spectrometers of a given hall. These spectrometers are also used in coincidence with temporarily installed "third-arm" detector packages such as specialized spectrometers or neutron detectors. Uses of such detectors have included measuring the deuteron tensor polarization in $(e, e d)[1]$, measurements of the neutron electric form factor $G_{E n}[2,3]$, and hypernuclear spectroscopy $\left({ }^{12} \mathrm{C}\left(e, e^{\prime} K^{+}\right)_{\Lambda}^{12} \mathrm{~B}\right)[4]$.

\section{CEBAF Large Acceptance Spectrome- ter - Hall B}

Hall B at JLab contains the CEBAF Large Acceptance Spectrometer, (CLAS), which can detect events over a large range of momentum, scattering angle and azimuthal angle. This large acceptance is particularly valuable when detecting multiparticle events where the particles are not strongly correlated with each other. The CLAS is a toroidal field spectrometer with six superconducting coils. It can detect positive and negative particles with scattering angles from $8^{\circ}$ to $140^{\circ}$ with a momentum resolution of $0.5 \%-1.0 \%$. The CLAS typically runs with an electron beam luminosity of $10^{34} \mathrm{~cm}^{-2} \mathrm{~s}^{-1}$.

With the Hall B tagger, the CLAS can utilize photon beams as well as electrons. The tagger tags Bremsstrahlung photons between $20 \%$ and $95 \%$ of the electron beam energy giving a photon energy resolution of $0.1 \%$. The tagger can operate with the CLAS at tagged photon rates of $1 \times$ $10^{7} \mathrm{~s}^{-1}$. Because of the low rate of tagged photon beams, the large acceptance of the CLAS makes it an ideal detector for tagged photon experiments.

As with the other halls, Hall B is host to specialized equipment beyond the standard detectors. A lead glass detector with over 600 blocks, used in conjunction with the tagger, has been installed to measure radiative decays of the $\phi$ meson [5]. Additional detectors will also be installed in order to measure the $\pi^{0}$ lifetime via the Primakoff Effect [6].

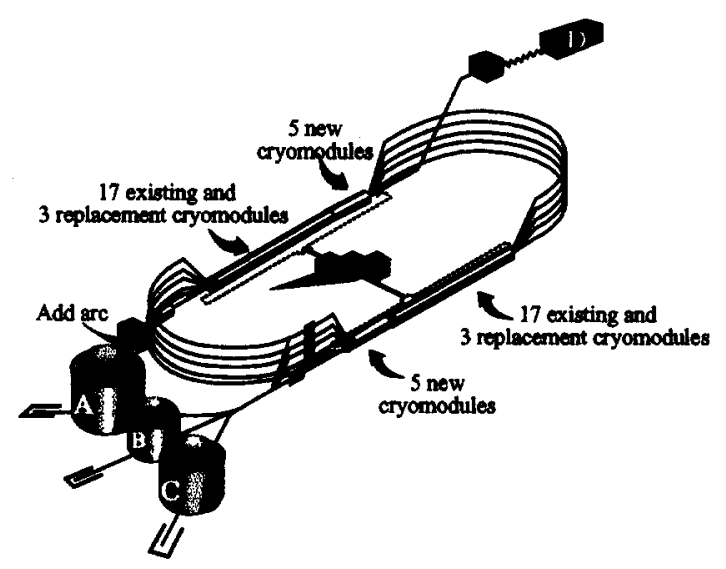

Figure 1. The Configuration for the proposed 12 $\mathrm{GeV}$ accelerator upgrade at JLab. New higher gradient cryomodules will be added and replace some existing cryomodules, delivering $11 \mathrm{GeV}$ beam to Halls A, B and C. An additional arc of magnets will bend the beam around for an additional pass of acceleration delivering $12 \mathrm{GeV}$ to the new Hall D. 


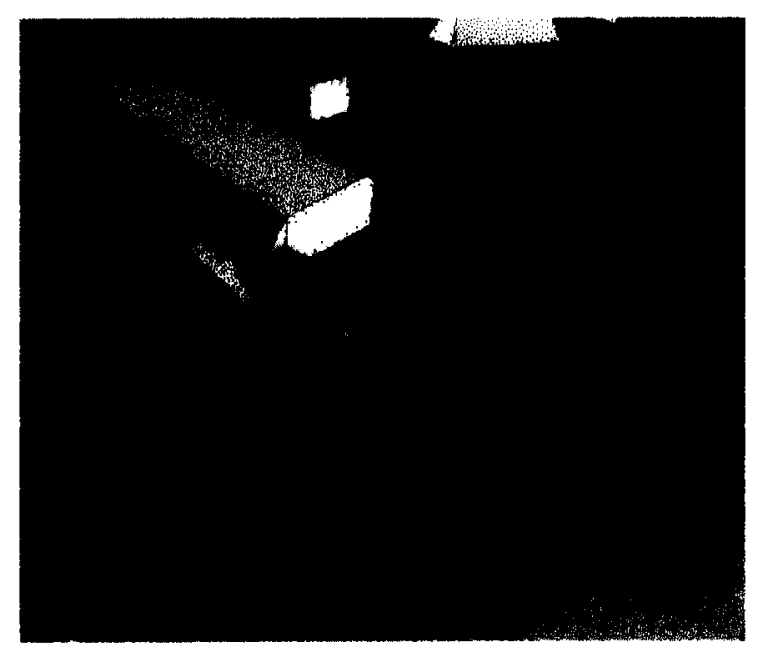

Figure 2. CAD drawing of the proposed $11 \mathrm{GeV} / c$ Super High Momentum Specterometer (SHMS) (left) in Hall $\mathrm{C}$ with the existing $7 \mathrm{GeV} / c$ High Momentum Spectrometer (right)

\section{Jefferson Lab Upgrade Plans}

Jefferson Lab is presently developing plans for a major upgrade of accelerator and experimental facilities. This upgrade will double the energy of the machine, providing a high current $11 \mathrm{GeV} / c$ electron beam to the existing end-stations and $12 \mathrm{GeV} / c$ beam to a new experimental area (Hall D). For Hall's A, B, and C, much of the existing experimental equipment will be retained and this equipment will be augmented with new spectrometers and detectors designed to exploit the new physics oportunities afforded by the higher beam energy. Hall D will consist of an all new facility for the GlueX [7] experiment.

The energy upgrade of the accelerator can be accomplished within the existing linac and recirculation arc tunnels. (See Fig. 1) Each linac tunnel contains 20 cryomodules, which provide 28 MV of acceleration each. Unused space in these tunnels allows the addition 5 more cryomodules of a new design giving up to $68 \mathrm{MV}$ each. In addition, 3 existing cryomodules in each arc will be replaced with modules of the newer design, giving per pass energy gains sufficient to supply $11 \mathrm{GeV}$ to the existing halls. In order to bend the higher energy beams, some of the magnets in the reciculation arcs will be replaced and a 10th arc of magnets will be added. This extra arc will bring the beam through the first linac for an extra acceleration pass before the new Hall $D$ which will receive a beam of $12 \mathrm{GeV}$.

One of the physics objectives of the Hall B upgrade plans is the study of Generalized Parton Distributions [8]. In order to carry out this and other physics programs at higher energies, the CLAS spectrometer will be upgraded to handle higher luminosities, have a more complete coverage of the final hadronic state and to extend the particle identification to higher momenta. The existing CLAS superconducting toroid will be retained, but many of the detector packages will be replaced allowing the device to be run at a luminosity of $10^{35} \mathrm{~cm}^{-2} \mathrm{~s}^{-1}$, about a factor of ten more than the current capability. The central detector and target will be moved upstream. This will allow for detection of smaller angle scattering and a more complete detection of the final state.

The Hall A upgrade will retain the two existing $4 \mathrm{GeV} / c$ spectrometers and add a new spectrometer. Part of the upgrade physics program for Hall $\mathrm{A}$ involves the measurement of spin structure functions in deep inelastic scattering. As luminosities with polarized targets are low, these measurements will be aided by the large solid angle of the proposed "Medium Acceptance Detector" (MAD). The MAD will have a solid angle of $15 \mathrm{msr}$ when operated at an $18.5^{\circ}$ scattering angle expanding to $30 \mathrm{msr}$ when at $35^{\circ}$ and above. This spectrometer will consist of two combined function dipole-quadrupole magnets with a central momentum of up to $6 \mathrm{GeV} / c$ and a momentum acceptance of $\pm 15 \%$.

The Hall C upgrade takes advantage of the existing HMS spectrometer that is already capable of $7 \mathrm{GeV} / c$ which will be complemented by constructing a new "Super High Momentum Spectrometer" (SHMS) with a maximum momentum of $11 \mathrm{GeV} / c$. (Fig. 2) For single particle detection, the SHMS will allow measurements at high $Q^{2}$ over a wide range of $x$. For SHMS in coincidence with the HMS, deep-exclusive scatter- 
ing (large $Q^{2}$ and small $t$ ) will be a major use of the upgraded hall. The SHMS design consists of two superconducting quadrupoles followed by a combined function dipole-quadrupole magnet and will have a momentum acceptance of up to $\pm 20 \%$. The minimum scattering angle will be $5.5^{\circ}$ where it will have a solid angle of $2 \mathrm{msr}$. At larger scattering angles, the solid angle can be increased to $4 \mathrm{msr}$ by moving the magnets and detector package closer to the target. This spectrometer, which will give momentum resolutions typically better than $1 \times 10^{-3}$, will have a flexible detector package to handle particle ID over the momentum range of the magnet.

The upgraded accelerator will deliver a $12 \mathrm{GeV}$ beam to Hall $\mathrm{D}$ where a diamond radiator will produce a high flux beam of linearly polarized photons. This tagged beam will be used by the GlueX experiment [7] to search for exotic mesons (quark-gluon hybrids). Mesons will be produced by photo-production on a hydrogen target. The mesons will be observed by detecting their decay products with detectors inside of a superconducting solenoid. The search for new and exotic mesons will be done by performing a Partial Wave Analysis on the data.

\section{JLab Research of Interest to Neutrino Physics}

The relationship between electron and neutrino scattering mechanisms and the overlap of JLab beam energies with many neutrino experiments suggests that comparison of electron scattering experiments to neutrino experiments can be fruitful.

The neutrino oscillation experiment at KEK/SuperK [9] uses a non-monochromatic beam of low energy neutrinos $\left(\left\langle E_{\nu}\right\rangle \approx\right.$ $1.3 \mathrm{GeV} / c)$. The neutrino oscillations are observed by measuring the neutrino flux and energy spectrum at two different distances from the neutrino source. This energy spectrum is measured primarily by observing $\left(\nu_{\mu}, \mu^{-}\right)$and $\left(\nu_{\mu}, \mu^{-} \pi\right)$ events in water. These events produce one and two Cerenkov light rings which are analyzed by comparing them to detailed Monte-Carlo simulations [10]. The Monte-Carlo simulations use models for $p\left(\nu_{\mu}, \mu^{-}\right)$and $p\left(\nu_{\mu}, \mu^{-} \pi\right)$ and use the Intra-Nuclear Cascade technique to simulate ${ }^{16} \mathrm{O}\left(\nu_{\mu}, \mu^{-}\right)$and ${ }^{16} \mathrm{O}\left(\nu_{\mu}, \mu^{-} \pi\right)$.

For electron scattering, $p\left(e, e^{\prime}\right)$ and $p\left(e, e^{\prime} \pi\right)$ probe the same nucleon structure as neutrino scattering and can be used to give guidance to neutrino Monte-Carlo codes in kinematics where neutrino data is lacking. Detailed deep inelastic electron scattering measurements, single arm measurements of the $F_{2}$ form factor and $R$, are ongoing at JLab [11]. The CLAS is measuring pion electroproduction, $p\left(e, e^{\prime} \pi^{+}\right) n$ and $p\left(e, e^{\prime} \pi^{0}\right) p$, over a wide kinematic range for beam energies between 2 and $4 \mathrm{GeV} / c$ [12]. Furthermore, Hall $\mathrm{C} p\left(e, e^{\prime} \pi^{+}\right)$experiments which measured the pion electromagnetic form factor [13] and longitudinal electroproduction of charged pions on hydrogen, deuterium and ${ }^{3} \mathrm{He}[14,15]$ could be of value.

Monte Carlo modeling of quasi-free $A\left(\nu_{\mu}, \mu^{-}\right)$ scattering is aided by comparison to quasi-elastic electron scattering, $A\left(e, e^{\prime}\right)$. JLab is engaged in a program of measurements of inclusive electron scattering on nuclei at $x>1$ kinematics [16]. While the focus of this research is on $x>1$, the data are taken across the quasi-elastic peak $(x<1$ and $x>1)$.

Simulations of $A\left(\nu_{\mu}, \mu^{-} \pi\right)$ depend in part on understanding pion transparency in nuclei. Pion transparency is essentially the probability that a pion produced inside of a nucleus will emerge from the nucleus relatively unscathed rather than being absorbed by the nucleus. A measurement of pion transparency is approved for Hall C [17]. This experiment will study transparency for pions with relatively large momenta $(\geq 2 \mathrm{GeV} / c)$, but not for the sub $\mathrm{GeV} / c$ pions that are relevant for neutrino experiments with several $\mathrm{GeV}$ beams. However, such measurements are quite feasible at JLab and could be proposed for future work and would help the modeling of $A\left(\nu_{\mu}, \mu^{-} \pi\right)$.

In many of the existing and potential experiments on nuclei described above, the measurements are inclusive; i.e. the full multiplicitiy of events is not detected. As many neutrino detectors are sensitive to multiple particle final states, neutrino Monte Carlo codes must simulate this multiplicity. These simulation codes could be 
modified to simulate $A\left(e, e^{\prime} X\right)$ reactions in the JLab CLAS detector. As the incident and scattered particle energies are precisely measured, a comparison of CLAS data to simulations could put strong constraints on the assumptions made by neutrino Monte Carlo codes. Data taken recently by the CLAS E2 group [18] (E2 is a group of seven JLab proposals that were run simultaneously) could be used in these comparisons. This data set includes various combinations of $1.1,2.2$, and $4.4 \mathrm{GeV}$ electron beams on ${ }^{3} \mathrm{He},{ }^{4} \mathrm{He}, \mathrm{CH}_{2}$, ${ }^{12} \mathrm{C},{ }^{56} \mathrm{Fe}$ targets.

Two of the experiments included in the E2 running involve the study of backward particle, including protons, production [19]. Comparing these measurements with observations of backward production of protons in neutrino scattering off of nuclear targets [20] could lead to a better understanding of the mechanisms of backward particle production.

\section{Conclusion}

JLab is $6 \mathrm{GeV}$ electron accelerator with an exciting research program that makes use of a set of complementary experimental facilities. The lab is currently preparing a proposal to double the energy of the accelerator and upgrade the experimental capabilities in order to further study nucleon/nucleus structure and interesting aspects of QCD.

There is an emerging interest in tying the field of neutrino physics to nuclear and high energy research with charged particle beams. The JLab energy range makes it well suited both to comparing electron and neutrino scattering research in order to improve nucleon/nuclear structure knowledge and to providing data which can help reduce uncertainties in neutrino Monte Carlo simulations. JLab welcomes participation in and proposals for research.

\section{Acknowledgements}

This work was supported by DOE Contract No. DE-AC05-84ER40150 under which the Southeastern Universities Research Association (SURA) operates the Thomas Jefferson National
Accelerator Facility (Jefferson Lab).

\section{REFERENCES}

1. D. Abbott et al., Phys. Rev. Lett. 84, 5053 (2000).

2. R. Madey et al., "Neutron Electric Form Factor via Recoil Polarimetry," Baryons 2002.

3. H. Zhu et al., Phys. Rev. Lett. 87, 081801 (2001).

4. L. Tang et al., AIP Conf. Proc. 603, 173 (2001).

5. R. T. Jones, AIP Conf. Proc. 432, 635 (1998).

6. JLab experiment E99-014.

7. A. R. Dzierba, C. A. Meyer, and E. S. Swanson, American Scientist 80, No. 5 (2000); http: //ww .gluex.org/.

8. A. V. Radyushkin, Nucl. Phys. A666, 225 (2000).

9. S. H. Ahn et al., Phys. Lett. B511, 178 (2001).

10. C. Mauger, Proc. of the 26th International Cosmic Ray Conference, (1999).

11. S. A. Wood, "Resonance region to DIS, quark-hadron duality at Jefferson Lab," these proceedings.

12. V. Burkert, "Electroexcitation of $N^{*}$ Resonances", Baryons 2002; H. Egiyan, to be published, Phys. Rev. D.

13. J. Volmer et al., Phys. Rev. Lett. 86, 1713 (2001).

14. D. Gaskell et al., Phys. Rev. Lett. 87, 202301 (2001).

15. D. Gaskell et al., Phys. Rev. C 65, 011001 (2002).

16. J. Arrington et al., Phys. Rev. Lett. 82, 2056 (1999); J. Arrington et al., Phys. Rev. C 64, 014602 (2001); JLab Experiment E02-019.

17. JLab experiment E01-107.

18. http://ww.jlab.org/ clase2/e2/ Run_Summary/RS_report.html.

19. JLab experiments E89-032 and E89-036.

20. M. Veltri, "A study of nuclear effects in $\nu$ interactions with the NOMAD detector," these proceedings.

21. JLab expriments E89-032 and E89-036.

22. JLab experiment E01-015. 